\title{
ON THE CLASS NUMBERS OF HILBERT CLASS FIELDS
}

\author{
FARSHID HAJIR
}

\section{In memory of Olga Taussky-Todd}

It is generally believed that a positive proportion of number fields (counted according to increasing absolute value of the discriminant), have class number one. This conjecture, and the numerical data that has led to it, have their origins in Gauss' Disquisitiones Arithmaticae, where it was conjectured that there are infinitely many quadratic fields with class number one, and that exactly nine of these are complex. While the second half of this statement was settled independently by Heegner [He] and Stark [St1], and was reduced to a finite computation by Baker $[\mathbf{B a}]$, the first half of it remains an important open problem. In fact, it is not known whether there exist infinitely many algebraic number fields with class number one. The modest aim of this note is to suggest another naturally arising family of number fields - namely the family of Hilbert class fields of imaginary quadratic fields - suspected of harboring infinitely many class number one fields, and of showing how this question is related to Gauss' conjecture. This family is also a natural testing ground for a proposed heuristic "principle," which in vague form reads: The Hilbert class field of a number field with cyclic class group tends to have class number one. We hope to test further and refine this admittedly imprecise statement in the future.

Let $K=K^{(0)}$ be an algebraic number field with class number $h$. The Hilbert class field of $K$, i.e. its maximal abelian unramified extension, has degree $h$ over $K$. A finite extension of $K$ with class number one (if it exists) must contain $K^{(1)}$ as a subfield. For $i=0,1,2, \ldots$, define $K^{(i+1)}$ to be the Hilbert class field of $K^{(i)}$. Define an invariant $\ell(K)$ called the "length of the Hilbert class field tower of $K$ " as follows: $\ell(K)$ is the smallest nonnegative integer $i$ such that $K^{(i)}=K^{(i+1)}$, if such an integer exists, and is $\infty$ otherwise. The latter possibility is equivalent to the statement that every finite extension of $K$ has class number greater than one. In 1964, Golod and Shafarevich [GS] proved that there exist number fields $K$ with $\ell(K)=\infty$. The Heegner-Stark-Baker result may be phrased as follows: there are only nine complex quadratic fields $K$ with $\ell(K)=0$. We conjecture that there are infinitely many complex quadratic fields $K$ with $\ell(K)=1$. 
The main results of the paper are: 1) with six exceptions, the complex quadratic fields $K$ with $\ell(K)=1$ have cyclic class group; and 2) if there are infinitely many complex quadratic fields $K$ with even class number and $\ell(K)=1$, then Gauss' conjecture is true. We also consider certain circumstances under which $\ell(K)>1$ even though the class group of $K$ is cyclic. It is not clear whether our conjecture will eventually shed any light on Gauss' conjecture, but the author hopes that it will broaden the methods of attack (most notably by the addition of techniques from complex multiplication) on the difficult problem of finding infinite families of number fields with small class number.

This paper has been organized in two parts. In the first half, the main results are proved, independent of any conjectures. In the second half, which is mostly devoid of proofs, we gather together heuristic arguments, as well as a small amount of numerical evidence, in support of the conjecture and "principle" introduced above.

\section{Main Results.}

1.1. $\ell(K)=1$.

We use the following notation throughout: For a number field $K, C l_{K}, h_{K}$, and $d_{K}$ are, respectively, its class group, class number, and discriminant. In this section, $K$ will always denote a complex quadratic field. We let $t_{K}$ be the number of distinct prime divisors of $d_{K}$. The focus of this section is to explore the consequences of

Conjecture. Infinitely many $K$ with $t_{K}=1$, as well as infinitely many $K$ with $t_{K}>1$, satisfy $\ell(K)=1$.

Some preliminary numerical data concerning this conjecture is collected at the end of the paper. Our main result can be summarized as follows.

Theorem 1. Suppose $\ell(K)=1$. Then $K$ is of one of the following types:

I. $t_{K}=1$, and $C l_{K}$ is a cyclic group (of odd order);

II. $t_{K}=2, C l_{K}$ is a cyclic group (of even order), and $d_{K}=d_{0} d_{1}$ where $d_{0}$ is the discriminant of a complex quadratic field with class number 1 and $d_{1}$ is either 8 or a prime number equal to the discriminant of a real quadratic field with class number 1.

III. $t_{K}=3, d_{K}$ is the product of three distinct integers, each of which is the discriminant of a complex quadratic field with class number 1.

Let us say that $K$ is of type I, II, or III, if it satisfies the corresponding condition stated above. Note that by Heegner-Stark-Baker, there are only finitely many fields of type III. In fact, the fields of type III with $\ell(K)=1$ 
all have class group of type $(2,2)$ and are listed in Yamamura [Ya1]: They are the fields of discriminant $-84,-132,-168,-228,-532,-627$. The following corollary shows that the above conjecture encompasses two betterknown ones.

\section{Corollary 2.}

1) If there are infinitely many $K$ satisfying $\ell(K)=1$, then there are infinitely many $K$ with cyclic class group.

2) If there are infinitely many $K$ with $t_{K}>1$ and $\ell(K)=1$, then there exist infinitely many real quadratic fields of prime discriminant with class number one.

Proof. 1) This is clear since the fields of type III are finite in number.

2) If $\ell(K)=1$ and $t_{K}>1$, then $K$ is of type II or III. There are finitely many $K$ of type III, therefore the assumption is that there exist infinitely many $K$ of type II. But if $K$ is of type II, then $d_{K}=d_{0} d_{1}$ where $d_{0}$ is one of the nine negative discriminants with class number one, and $d_{1}$ is the (prime) discriminant of a real quadratic field with class number one. This proves $2)$.

Remark. According to the Cohen-Lenstra heuristics [CL], 97.7 $\cdots \%$ of the fields $K$ with $t_{K}=1$ or 2 have cyclic class group, and $75.4 \cdots \%$ of the real quadratic fields of prime discriminant have class number one.

The proof of the Theorem is facilitated by the following two lemmas.

Lemma 3. Suppose $\ell(K)=1$. Then every quadratic subfield of $K^{(1)}$ other than $K$ has class number 1.

Proof. If $t_{K}=1$, then the only quadratic subfield of $K^{(1)}$ is $K$, so we may assume $t_{K}>1$. Suppose $K_{0}$ is a quadratic subfield of $K^{(1)}$ distinct from $K$; then $L=K K_{0}$ is an intermediate subfield of $K^{(1)} / K$, hence quadratic unramified over $K$. Since $L$ is a $(2,2)$-extension of $\mathbb{Q}$, there is a third quadratic subfield $K_{1}$ of $L$. Let $h_{0}, h_{1}$ be the class number of $K_{0}, K_{1}$, respectively. By a classic result of Herglotz $[\mathbf{H}]$, the class number of $L$ is given by

$$
h_{L}=\frac{h h_{0} h_{1}}{\delta}
$$

where $\delta=1$ or 2 . The Hilbert class field of $L$ is an abelian unramified extension of $K^{(1)}$ and is consequently $K^{(1)}$ itself since this field has class number 1 . Therefore, $h_{L}=h / 2$, so by Herglotz, $h_{0} h_{1}=\delta / 2$. We see then that $\delta=2$ and $h_{0} h_{1}=1$, proving the lemma. 
Lemma 4. Suppose $\ell(K)=1$. Then the odd part of $C l_{K}$ is cyclic. If the 2-part of $C l_{K}$ is not cyclic, then $d_{K}$ is the product of discriminants of three distinct complex quadratic fields with class number 1.

Proof. It is well known (see, Bond [Bo] or Nomura [No]) that if the class group of $K$ has non-cyclic $p$-part for an odd prime $p$, then $p$ divides the class number of the Hilbert $p$-class field of $K$. This is not always true for $p=2$, however: The fields of type III and $\ell(K)=1$ listed above are examples. Assume the class group of $K$ has non-cyclic 2-part and that the Hilbert class field of $K$ has class number one. By genus theory, the discriminant $d$ of $K$ is divisible by at least three primes. Write $d=d_{1} d_{2} d_{3}$ where $d_{j}$ $(j=1,2,3)$ is the discriminant of a quadratic field. Then $L_{j}=K\left(\sqrt{d_{j}}\right)$ is an unramified quadratic extension of $K$ for $j=1,2,3$. It follows from Lemma 3 that the six quadratic fields

$$
\mathbb{Q}\left(\sqrt{d_{j}}\right), \mathbb{Q}\left(\sqrt{d_{k} d_{l}}\right), \quad j, k, l \in\{1,2,3\}, k \neq l,
$$

all have class number one. Therefore, $\mathbb{Q}\left(\sqrt{d_{k} d_{l}}\right)$ must be a real quadratic field for $k \neq l$ (otherwise, the class number of this field would be even). Hence, $d_{1}, d_{2}, d_{3}$ must have the same sign. Since $d$ is negative, $d_{1}, d_{2}, d_{3}$ must all be negative. Therefore they are to be found in the list of nine negative fundamental discriminants with class number one.

Proof of Theorem 1. Suppose $\ell(K)=1$. If $t_{K}=1$, then by genus theory and Lemma $4, C l_{K}$ is cyclic of odd order, hence $K$ is of type I. By the same reasoning, if $t_{K}=2$, then $C l_{K}$ is cyclic of even order; we must have $d_{K}=$ $d_{0} d_{1}$ where $d_{0}$ is a negative prime discriminant, and $d_{1}$ is a positive prime discriminant. Furthermore, by Lemma 3, the quadratic fields of discriminant $d_{0}$ and $d_{1}$ have class number 1, i.e. $K$ is of type II. Finally, if $t_{K} \geq 3$, then the 2-part of $C l_{K}$ is not cyclic, hence by Lemma $4, K$ is one of the finitely many fields of type III.

As a final illustration of the uses of Herglotz' formula, we give a short proof of the following well-known result (see e.g. Bond [Bo]). For a prime $p$, the Hilbert $p$-class field of $K$ is the maximal $p$-extension $K_{p}^{(1)}$ contained in $K^{(1)}$.

Proposition 5. Suppose the Hilbert 2-class field of $K$ has odd class number. Then $t_{K} \leq 3$.

Proof. Suppose $t_{K}>3$. Write $d_{K}=d_{0} d_{1} d_{2} d_{3}$ where the $d_{j}$ are fundamental discriminants. Since the Hilbert 2-class field of $K$ has odd class number, it 
is also the Hilbert 2-class field of the quadratic unramified extension $L=$ $K\left(\sqrt{d_{0}}\right)$. For a number field $F$, let $h_{F}^{\prime}$ be the 2-part of the class number of $F$. Then, by Herglotz,

$$
h_{L}^{\prime}=\frac{1}{\delta} h_{K}^{\prime} h_{\mathbb{Q}\left(\sqrt{d_{0}}\right)}^{\prime} h_{\mathbb{Q}\left(\sqrt{d_{1} d_{2} d_{3}}\right)}^{\prime}=\frac{1}{2} h_{K}^{\prime} .
$$

It follows that $\mathbb{Q}\left(\sqrt{d_{1} d_{2} d_{3}}\right)$ has odd class number, a contradiction.

1.2. $\ell(K)>1$.

As mentioned above, even though one expects that the fields of type I and type II are each infinite in number, it is not even known whether there are infinitely many complex quadratic fields with cyclic class group. (Indeed, the author is not aware of a proof that there are infinitely many algebraic number fields with cyclic class group.) It is not difficult to see that there exist fields $K$ of each type I, II and III, with $\ell(K)>1$ (see e.g. the tables in Yamamura [Y2]). One might ask whether there are infinitely many complex quadratic fields $K$ of type I (respectively, of type II) with $\ell(K)>1$. As an interesting special case of this question, consider fields of type I whose class number is divisible by 3 ; for these fields, the parity of $h_{K^{(1)}}$ is influenced by the existence of certain quartic fields, as we now explain.

Proposition 6. Suppose $K=\mathbb{Q}(\sqrt{-p})$, where $p \equiv 3(\bmod 4)$ is a prime number. There exists a quartic field of discriminant $-p$ if and only if $K$ admits a cyclic cubic unramified extension whose class number is even.

Sketch of Proof. It is not difficult to show (see, e.g., Kondo [Ko]) that the splitting field $E$ of a quartic field $F$ of discriminant $-p$ is an $S_{4}$-extension of $\mathbb{Q}$, unramified over $K$; since $E / K$ has Galois group $A_{4}$, it admits an intermediate field $N$ such that $N / K$ is cyclic cubic (hence 3 divides $h_{K}$ ) and $E / N$ is $(2,2)$, hence $h_{N}$ is even. Conversely, suppose there exits a cyclic cubic extension $N / K$ such that $N$ has even class number. By Lemma 8, the class group of $N$ has 2-rank at least 2, hence, there is an unramified extension $E$ of $K$ with absolute Galois group $S_{4}$, proving the existence of the quartic field $F$. See Serre [Se] for examples and a discussion of the associated two-dimensional Galois representations.

There are six examples of this phenomenon for $K=\mathbb{Q}(\sqrt{-p})$ with $p<$ 1000 , namely $p \in\{283,331,491,563,643,751\}$. In each of these cases, we have $3 \mid h_{K}$ and $h_{N}$ is even (hence divisible by 4 ), where $N$ is the unique unramified cyclic cubic extension of $K$. The field $N_{2}^{(1)}$ is a normal $S_{4}$-extension of $\mathbb{Q}$ and admits a quartic subfield with discriminant $-p$. It seems plausible, 
though it is probably difficult to prove, that there are infinitely many quartic fields with prime discriminant $-p$; if, in addition, for infinitely many of these primes, $\mathbb{Q}(\sqrt{-p})$ has cyclic class group, then there are infinitely many type I fields with class field tower of length greater than one. This question on quartic fields is also related to the question of the existence of infinitely many non-isomorphic elliptic curves over $\mathbb{Q}$ with prime conductor.

As for fields of type II, one expects that there are infinitely many real quadratic fields with prime discriminant and class number one which admit unramified non-solvable extensions (see, for example, [Ya2] and its references), hence infinitely many type II fields $K$ with $\ell(K)>1$.

\section{Heuristics.}

\subsection{Cyclic class groups.}

This section contains an informal discussion of some heuristic principles and numerical data concerning class numbers, which provide some justification for introducing the conjecture discussed in the first half of the paper.

We can identify at least three phenomena responsible for the existence of number fields with large class number: (a) genus theory; (b) CM extensions; (c) non-cyclic class groups. We give a brief explanation for each.

(a) If $K / F$ is a Galois extension of prime degree $p$ in which many primes ramify, then the $p$-part of the class group of $K$ has large $p$-rank (e.g. Martinet [Ma]), and therefore $K$ has large class number.

(b) Stark [St2] has shown that CM fields tend to have large class number: As $K$ runs over totally complex quadratic extensions of a fixed totally real field $F$, the "minus class number" $h_{K} / h_{F}$ tends to infinity. In fact, under either the Artin Conjecture or the Generalized Riemann Hypothesis, there are only finitely many CM fields of given minus class number; see Odlyzko [Od] where weaker unconditional statements are also proved.

(c) The mechanism behind large class numbers in (a) and (b) is ramification, but sometimes class numbers are large as a result of non-cyclic unramified extensions; for instance, if $F$ is a number field satisfying the Golod-Shafarevich criterion at $p$ (namely, the $p$-rank of $C_{F}$ is large with respect to the degree of $F$ ), then the $p$-rank of $C l_{K}$ tends to infinity as $K$ runs over the unramified $p$-extensions of $F$ [Ha2].

On the other hand, the only method known to the author for bounding class numbers from above is that of discriminant bounds, which applies only in a limited number of cases. In the face of the numerical data suggesting that class number one is a common occurrence, but lacking an understanding of the mechanisms behind it, one can gather together the known mechanisms responsible for large class numbers and hypothesize that in their absence, 
class numbers tend to be small.

In an attempt to formulate a more precise statement, let us note that sometimes a number field has large class number because one of its subfields is under the influence of one of the phenomena (a), (b), or (c) discussed above. To take this into account, for a number field $K$, define the "old class field" $K_{\text {old }}^{(1)}$ of $K$ as follows:

$$
K_{\mathrm{old}}^{(1)}=\bigcup_{K_{0} \subset K} K_{0}^{(1)}
$$

i.e. $K_{\text {old }}^{(1)}$ is the composita of the Hilbert class fields of all proper subfields of $K$. Clearly, we have $K_{\text {old }}^{(1)} \subseteq K^{(1)}$. Define the "new class number" of $K$ to be $h_{K}^{\text {new }}=\left[K^{(1)}: K_{\text {old }}^{(1)}\right]$.

Following Stark, we can formulate the following hypothesis: In a "generic" family of non-CM number fields, ordered according to increasing absolute value of discriminant, a positive proportion of the fields have new class number one. Of course, it is difficult to make the term "generic" more precise, but, for instance, for the fields in question, normal subextensions of prime index should have few ramifying primes (in order to avoid class number contributions coming from genus theory).

Now we would like to suggest that one such "generic" family is that of Hilbert class fields of number fields with cyclic class group. In other words,

(1) A positive proportion of number fields $K$

with cyclic $C l_{K}$ have $\ell(K)=1$.

Some corroborating evidence for this heuristic principle is provided by

Lemma 7. If the $p$-class group of $K$ is cyclic, then $p$ does not divide the class number of the Hilbert $p$-class field of $K$.

Proof. This well known fact can be proved as follows. If $G$ is the Galois group, over $K$, of the maximal unramified $p$-extension of $K$, then, by the Burnside basis theorem, $G$ is cyclic because its maximal abelian quotient is isomorphic to $C l_{K}$ by class field theory. Since $G$ is abelian, the Hilbert $p$-class field of $K$ is the maximal unramified $p$-extension of $K$, so it must have class number prime to $p$.

The principle (1) is simplest to study and most effective when $C l_{K}$ has prime order. Let us assume then that $K$ is a number field with prime class 
number $p$. Then $p$ does not divide $h_{K^{(1)}}$. Of course, some other prime $q$ might divide $h_{K^{(1)}}$, but this has strong consequences:

Lemma 8. Suppose $L$ is a Galois extension of $K$ of prime degree $p$, and that $q$ is a prime not dividing $p h_{K}$. If $q$ divides $h_{L}$, then $q^{f}$ divides $h_{L}$, where $f$ is the order of $q$ in $(\mathbb{Z} / p \mathbb{Z})^{\times}$. Moreover, the q-rank of $C_{L}$ is divisible by $f$.

Proof. This follows easily from a study of the $q$-part of $C_{L}$ as $\operatorname{Gal}(L / K)$ module; see, e.g., Iwasawa [Iw].

Returning to our $K$ with class number $p$, we see that $h_{K^{(1)}}$, if it is not 1 , is a product of prime powers which are congruent to 1 modulo $p$, hence tends to be quite a bit larger than $p$, especially for most large $p$. Moreover, if $q$ divides $h_{K^{(1)}}$ and $q \not \equiv 1(\bmod p)$, then $C l_{K^{(1)}}$ not cyclic. This is very significant because a principle of Lenstra $[\mathrm{CL}]$ asserts that when predicting the proportion of number fields that have a given abelian group $G_{2}$ as their class group, one should weigh the group with the factor $1 / \mid$ Aut $G \mid$. In particular, the components of class groups of number fields not under the influence of genus theory tend to be cyclic. For example, primes $q$ which are primitive roots $\bmod p$ are not very likely to divide $h_{K^{(1)}}$. These restrictions on the shape of the class group of $K^{(1)}$ suggest that this field ought to have an affinity for having class number 1 . When $K^{(1)}$ has a non-trivial prime factor $q$, then the residue class of $q(\bmod p)$ is likely to be 1 , since this would maintain the possibility that $C l_{K^{(1)}}$ is cyclic. In this case, we have the following result, due to Honda [Ho].

Proposition 9 (Honda). Suppose $h_{K}=p$ is prime, and $K^{(1)}$ has cyclic class group. If $h_{K^{(2)}}>1$, then $C l_{K^{(2)}}$ is the direct sum of $p$ copies of an abelian group.

In summary, a large positive density of number fields $K$ with prime class number should satisfy $\ell(K)=1$, with most of the others satisfying $\ell(K)=2$.

For number fields with cyclic class group of composite cardinality, one would still expect that the class number of the Hilbert class field is often one. Take any prime $p$ dividing $h_{K}$ and consider the abelian unramified extension $K^{(1)} / K_{p}^{(1)}$, where $K_{p}^{(1)}$ is the Hilbert $p$-class field of $K$. Since $p$ does not divide the class number of $K_{p}^{(1)}$, Lemma 8 and the Lenstra heuristic imply that $h_{K^{(1)}}$ tends to be prime to $p$. The possibility exists that for a different prime $q$ dividing $h_{K}$, the power of $q$ in the class number grows in going from $K$ to $K_{p}^{(1)}$, but this also figures to be a relatively rare occurence, because it transfers to the extension $K^{(1)} / K_{q}^{(1)}$, where the class number of the base field and the degree of the extension are prime to $q$, so Lemma 8 applies. 


\subsection{Real quadratic fields.}

There are examples where the heuristic principle (1) reduces to other, more tested and better established, ones. Consider, for instance, real quadratic fiels $K$ of discriminant $5 p_{1}$, where $p_{1} \equiv 13,17(\bmod 20)$ is a prime. It is easy to see that the 2-class group of $K$ has order 2 (since $p_{1}$ is a non-residue mod 5), and, by [CL], one expects infinitely many of these $K$ to have trivial odd-class group. For those $K$, we have $K^{(1)}=\mathbb{Q}\left(\sqrt{5}, \sqrt{p_{1}}\right)$; by Lemma 7 , $K^{(1)}$ has odd class number, hence by Herglotz, $h_{K^{(1)}}=h_{\mathbb{Q}(\sqrt{5})} h_{\mathbb{Q}\left(\sqrt{p_{1}}\right)}$. Thus, $\ell(K)=1$ if and only if $h_{\mathbb{Q}\left(\sqrt{p_{1}}\right)}=1$, which we expect to happen infinitely often, again, by [CL]. More generally, the Cohen-Lenstra heuristic (which incidentally fits very well with the best available numerical data) predicts that a large positive density of real quadratic fields of class number 2 have Hilbert class field tower of length 1 .

\subsection{Complex quadratic fields.}

Now suppose $K$ is complex quadratic and $L$ is an unramified extension of $K$ of odd prime degree $p$. Then $L$ is a dihedral extension of $\mathbb{Q}$; it has a subfield of index 2 , call it $F$, which has a unique real place, and is a non-Galois degree $p$ extension of $\mathbb{Q}$.

Lemma 10. With $L, K, F$ as above, we have $h_{L}=h_{F}^{2} h_{K} a / p$, where a is either 1 or $p$. If the p-part of $C l_{K}$ is cyclic, then $a=1$.

Proof. The first statement is from Moser [Mo, Theorem IV.1]. If the $p$-part of $C l_{K}$ is cyclic, then it follows from Lemma 7 that the $p$-part of the class number of $L$ is $h_{K} / p$. Therefore, a cannot be equal to $p$.

In particular, if $K$ is complex quadratic with prime class number $h_{K}=p$, then $h_{K^{(1)}}=h_{F}^{2}$ where $F$ is a maximal real subfield of $K^{(1)}$. Since $F$ has no proper subfields, its class number is its new class number, and $F$ is not $\mathrm{CM}$, of course. Furthermore, $p$ does not divide $h_{K^{(1)}}$ nor $h_{F}$. The Stark principle, then, suggests that $h_{F}$ and $h_{K^{(1)}}$ are 1 frequently. Note also that Stark's principle suggests that complex quadratic $K$ with $\ell(K)=1$ do not have CM subfields of large degree, which, by genus theory, translates into the condition that the 2-class group ought to be cyclic; this was confirmed in Theorem 1.

Lastly, let us consider some numerical data regarding complex quadratic fields. Much useful data (some of it conditional upon the Generalized Riemann Hypothesis) about the maximal unramified extension of complex quadratic fields of discriminant $-d$ with $0<d<1000$ is compiled in Yamamura [Ya2]. In this range, there are 239 fields with cyclic class group. These can be partitioned into 3 sets: There are 89 fields of type I, 109 fields of 
type II, and 41 remaining fields of neither type. The latter 41 fields $K$ have $\ell(K)>1$ by Theorem 1 . Of the 89 fields of type I, exactly 6 have $\ell(K)>1$ (they are the fields that admit unramified $A_{4}$-extensions; we met them in Section 1.2). Finally, all but one $(d=731=17 \cdot 43)$ of the 109 fields of type II have $\ell(K)=1$. Overall, 48 (approximately 20\%) of the 239 fields with cyclic class group have $\ell(K)>1$. Moreover, for $K$ of discriminant $-d$ with $0<d<15000$ and odd prime class number $\leq 19$, the 2-part of the class number of $K^{(1)}$ is trivial with four exceptions (three we met in Section 1.2, and $d=14947)$. This was checked using elliptic units in [Ha1].

\section{References}

[Ba] Baker, Linear forms in the logarithms of algebraic numbers, Mathematika, 13 (1966), 204-216.

[Bo] R. Bond, Unramified abelian extensions of number fields, J. Number Theory, 30 (1988), 1-10.

[CL] H. Cohen and H.W. Lenstra, Heuristics on class groups of number fields, Lecture Notes in Math., 1068 (1984) 33-62.

[GS] E. Golod and I. Shafarevich, On class field towers, Izv. Akad. Nauk SSSR, 28 (1964), 261-272 [In Russian]; English transl. in 'Amer. Math. Soc. Transl.,' Vol. 48, pp. 91-102, American Math Soc., Providence, R.I., 1965.

[Ha1] F. Hajir, Unramified elliptic units, Thesis, MIT, 1993.

[Ha2] - On the growth of p-class groups in p-class field towers, J. Algebra, 188 (1997), 256-271.

[He] K. Heegner, Diophantische analysis und modulfunktionen, Math. Z., 56 (1952), 227-253.

[H] G. Herglotz, Über einen Dirichletschen Satz, Math. Z., 12 (1922), 255-261.

[Ho] T. Honda, On absolute class fields of certain algebraic number fields, J. Reine Angew. Math., 203 (1960), 80-89.

[Iw] K. Iwasawa, A Note on ideal class groups, Nagoya Math J., 27 (1966), 239-247.

[Ko] T. Kondo, Algebraic number fields with discriminant equal to that of a quadratic number field, J. Math. Soc. Japan, 47 (1995), 31-36.

[Ma] J. Martinet, Tours de corps de classes et estimations de discriminants, Inv. Math., 44 (1978), 65-73.

[Mo] N. Moser, Unités et nombre de classes d'une extension galoisienne diédrale de $\mathbb{Q}$, Abh. Math. Sem. Univ. Hamburg, 48 (1979), 54-75.

[No] A. Nomura, On the existence of unramified p-extensions, Osaka J. Math., 28 (1991), 55-62.

[Od] A.M. Odlyzko, Some analytic estimates of class numbers and discriminants, Invent. Math., 29 (1975), 275-286.

[Se] J.-P. Serre, Modular forms of weight 1 and Galois representations, in 'Algebraic number fields,' (A. Fröhlich, ed.) Academic Press, London, (1977), 193-268.

[St1] H.M. Stark, A complete determination of the complex quadratic fields of class- 
number one, Mich. Math. J., 14 (1967), 1-27.

[St2] Some effective cases of the Brauer-Siegel theorem, Invent. Math., 23 (1974), 135-0152.

[Ya1] K. Yamamura, The determination of the imaginary abelian number fields with class number one, Math. Comp., 62 (1994), 899-921.

[Ya2] _ Maximal unramified extensions of imaginary quadratic number fields of small conductors, preprint, 1997.

UCLA

Los Angeles CA 90095

E-mail address: fhajir@math.ucla.edu

AND

California State University, San Marcos

SAN MARCOS CA 92096

E-mail address: fhajir@csusm.edu 